\title{
Behavioural and clinical factors associated with depression among individuals with diabetes
}

Z. Yekta, ${ }^{7}$ R. Pourali ${ }^{7}$ and R. Yavarian ${ }^{2}$

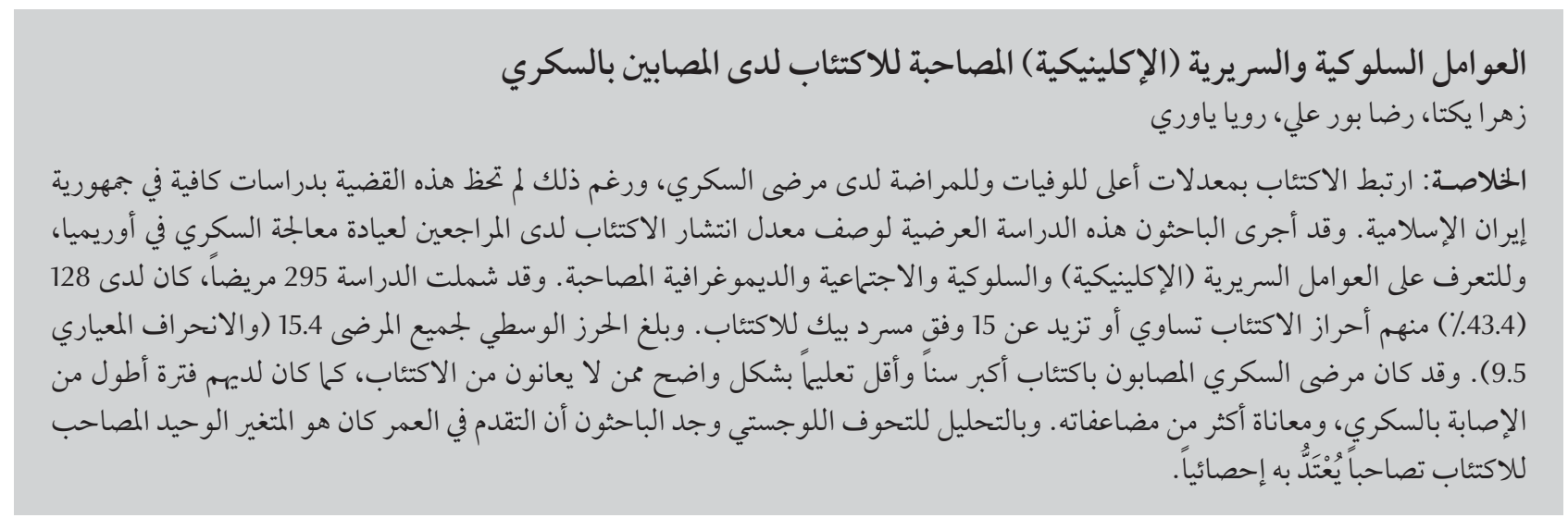

ABSTRACT Depression has been linked to greater mortality and morbidity in diabetic patients, but this issue has not been adequately studied in the Islamic Republic of Iran. This cross-sectional study described the prevalence of depression in patients attending a diabetes clinic in Urmia and determined the associated sociodemographic, behavioural and clinical factors. Of 295 patients, 128 (43.4\%) had depression scores $(\geq 15)$ on the Beck Depression Inventory. The mean score for all patients was 15.4 (SD 9.5). Those with depression were significantly older and less educated than those without depression, had a longer duration of diabetes and were more likely to suffer complications. On logistic regression analysis, older age was the only variable significantly associated with depression.

\section{Facteurs comportementaux et cliniques associés à la dépression chez les individus diabétiques}

RÉSUMÉ Une relation a été établie entre une mortalité et une morbidité plus fortes chez les patients diabétiques, mais le sujet n'a pas été correctement étudié en République islamique d'Iran. Cette étude transversale décrivait la prévalence de la dépression chez les patients suivis dans une clinique du diabète à Urmia et définissait les facteurs socio-démographiques comportementaux et cliniques qui y sont associés. Sur 295 patients, 128 (43,4 \%) avaient un score de dépression ( $\geq 15$ ) sur l'Inventaire de la dépression de Beck. Le score moyen pour l'ensemble des patients était 15,4 (écart type 9,5). Les patients souffrant de dépression étaient nettement plus âgés et moins éduqués que ceux qui n'en souffraient pas, étaient diabétiques depuis plus longtemps et présentaient davantage de risques de complications. À l'analyse de régression logistique, l'âge était la seule variable significative associée à la dépression. 


\section{Introduction}

Depression is recognized as an important co-morbidity in a number of chronic medical conditions such as diabetes [1]. Reports indicate that patients with diabetes are 1.5-2 times more likely to have depression than those without [1]. A recent meta-analysis of 39 studies of patients with diabetes estimated the prevalence of major depression (based on psychiatric interviews) as $11 \%$ and elevated depression symptoms (based on depression rating scales) as $31 \%$ [2]. Depression contributes to poor metabolic control, decreased quality of life and increased medical morbidity and mortality in patients with diabetes [2-5], as well as significantly higher medical costs [6]. More encouragingly, however, there is evidence that therapy to treat depressive conditions is effective and improves the mood, functioning and quality of life in diabetic patients $[7,8]$.

Some sociodemographic, behavioural and clinical factors seem to be associated with depression in diabetic patients although the results are conflicting. Research has shown a high risk for female sex, younger age, lower education and lower income [2]. Another study indicated that age and chronic conditions may be significant independent predictors of depressive symptoms, but that depression was unrelated to sex, ethnic group, duration or type of diabetes [9].

Despite the many reports of an association between depression and diabetes, the validity of these findings in different cultures and communities remains to be shown [10]. Diabetes currently affects approximately $3 \%-5 \%$ of the population of the Islamic Republic of Iran and this figure is expected to rise considerably by the year 2025 [11]. Furthermore, the Global Burden of Disease Survey estimated that by 2020 major depression will be second leading cause of disease burden worldwide after ischaemic heart disease [12].

In the Islamic Republic of Iran research on depression in diabetic patients is lacking, and depression frequently remains unrecognized and undertreated. The aim of this study was to describe the prevalence of depression in individuals with diabetes in Urmia, Islamic Republic of Iran, and determine the associated sociodemographic, behavioural and clinical factors.

\section{Methods}

This was a cross-sectional study conducted from February to August 2006.

\section{Sample}

A convenience sample was drawn from attendees at the Taleghani diabetes clinic in Urmia, Islamic Republic of Iran. Based on a power analysis using a moderate effect size and probability level of 0.05 , significance level of 0.05 and 0.80 power, a sample size of 300 participants was judged to be adequate.

Physicians referred patients for participation in the study based on the following criteria: currently taking any diabetic medication, fasting blood glucose $>126 \mathrm{mg} / \mathrm{mL}$ (confirmed with a second out-of-range test); and a hospital discharge diagnosis of diabetes. Patients with a current diagnosis of type 2 diabetes and age $>30$ years were assigned consecutively to the study. Patients diagnosed with type 1 diabetes (onset $<30$ years of age and insulin as the first treatment prescribed) were excluded [2].

The aim of the study was explained to all participants, who signed the consent form. Permission to conduct this research was granted by the ethics committee at the Urmia Medical Sciences University. Only 5 patients refused to participate and therefore 295 patients were enrolled in this study.

\section{Data collection}

A questionnaire was used to collect data about clinical status: age at onset and duration of diabetes; complications of diabetes; treatment intensity; current smoking (daily and occasional smokers); and body mass index (BMI). Treatment intensity was classified into: insulin therapy (alone or combination) or other therapy (oral agents, diet). BMI was divided into 2 categories: normal $<25 \mathrm{~kg} / \mathrm{m}^{2}$ or overweight $\geq 25 \mathrm{~kg} / \mathrm{m}^{2}$. Demographic data were also collected about: age, sex, marital status, educational level and income level. Marital status was classified as unmarried (single/divorced/widowed) or married. Participants were classified into low or middle/high income groups based on total household income divided by the number of household members. This categorization was based on patients' reports. Educational level was classified as low (illiterate/primary/middle school) or high (high school/college/ university).

All patients completed a Farsi version of the Beck Depression Inventory (BDI) [13]. The BDI is a standard selfreported questionnaire containing 21 items that measure the presence and severity of cognitive and somatic symptoms of depression on a scale from 0-63, with higher scores indicating greater depression. Each item evaluates a category according to a scale of 4 possible responses of increasing severity. It has been validated in patients with diabetes and screens effectively for major depression in this population $[3,14,15]$. Standard cut-offs were used in this study: $0-15$ (no depression); 16-30 (mild), 31-46 (moderate) and $\geq 47$ (severe).

All information was collected by interview. Two trained medical students interviewed the patients and filled out the forms. They also helped patients to fill out the BDI if they had any problems. 


\section{Analysis}

All statistical analyses were done using SPSS, version 12.0. The prevalence was determined by simple percentages. We also examined group differences in sociodemographic, behavioural and clinical variables between patients with and without depression by using the chi-squared test for categorical variables and independent $t$-test for continuous data. The effect of variables on depression was also estimated in men and women separately. The $P$-value was significant at $<0.05$.

Logistic regression models were constructed to model the odds of having depression versus no depression. All variables were examined for the association with depression including sociodemographic factors (age, sex, education, marital status and income), behavioural risk factors (BMI and smoking) and diabetes clinical factors (duration of diabetes, treatment intensity and number of complications). Odds ratios (OR) and their $95 \%$ confidence intervals (CI) were estimated for all variables in the models.

\section{Results}

\section{Background characteristics}

The mean age of this sample of diabetic patients was 52.4 [standard deviation (SD) 12.0] years. Of the 295 patients 205 (69.5\%) were women. The mean age of males and females was not significantly different [51.6 (SD 13) years versus 53.5 (SD 12) years]. Three-quarters $(74.8 \%)$ of participants had a low education level, below high school. The sample was predominantly low income with $55.9 \%$ of participants reporting an income < US\$ 150 per month.

The mean BMI was 28.4 (SD 4.9) $\mathrm{kg} / \mathrm{m}^{2}$, and $78.2 \%$ of participants were overweight (BMI $\left.\geq 25 \mathrm{~kg} / \mathrm{m}^{2}\right)$. Treatment intensity showed that $83.0 \%$ were managed with oral agents/diet only and $17.0 \%$ were on insulin (alone or combination). Half of patients (50.9\%) reported having at least 1 complication of diabetes, e.g. cardiovascular disease, nephropathy, retinopathy and diabetic foot; $10.1 \%$ experienced 2 or more complications. There were higher rates of cardiovascular disease and nephropathy compared with other complications. Patients with 1 or more complication had a significantly higher mean duration of diabetes than those without any complications [9.4 (SD 6.8) years versus 7.3 (SD 6.0) years] $(P<0.005)$ and a significantly higher mean age $[56.0$ (SD 13) years versus 47.5 (SD 12) years] $(P$ $<0.001$ ).

\section{Prevalence of depression}

Of the 295 patients, 128 (43.4\%) met the diagnostic criteria for depression (BDI score $\geq 15$ ); $36.3 \%$ were classified as minor (BDI score 16-30), 5.8\% as moderate (BDI score 31-46) and $1.3 \%$ as severe depression (BDI score $\geq$ 47). The mean score on the BDI for the whole sample of diabetic patients was 15.4 (SD 9.5).

\section{Factors associated with depression}

Compared with the group without depression, those with depression were significantly older [mean age 55.6 (SD 12) years versus 50.4 (SD 13) years] $(P$
$<0.05$ ) (Table 1). Patients who were $\geq$ 50 years had a significantly higher risk of having depression symptoms than those $<50$ years (OR 1.64, 95\% CI: $1.21-2.42)(P<0.01)$.

More women (94/205, 45.9\%) than men $(34 / 90,37.8 \%)$ had depression scores on the BDI but this was not statistically significant. However, mean BDI scores for women were significantly higher than for men [16.6 (SD 10.4) versus 12.8 $(S D$ 8.1 $)](P<0.02)$.

Depressed patients were less educated than nondepressed patients (only $81.3 \%$ versus $68.3 \%$ had low educational level $)(P<0.05)$ and poorer $(64.1 \%$ versus $52.4 \%$ had low income level) $(P<0.05)$ (Table 1). There was also significant difference regarding marital status; a higher proportion of depressed patients were married (97.4\%) than nondepressed patients $(92.9 \%)(P<$ 0.05).

Clinical factors associated with depression were duration of diabetes and having complications of diabetes. The mean duration of diabetes was 9.2 (SD 6.8 ) years in depressed and 7.8 (SD 5.1) years in nondepressed patients $(P$ $<0.05$ ). Other clinical and behavioural factors (treatment intensity, smoking, overweight) had the same distribution in depressed and nondepressed diabetic patients (Table 1).

\begin{tabular}{lccc}
\hline $\begin{array}{l}\text { Table 1 Demographic, behavioural and clinical features of diabetic patients with } \\
\text { and without depression scores on the Beck Depression Inventory (BDI) }\end{array}$ \\
\hline $\begin{array}{lccc}\text { Vo depression } \\
(\boldsymbol{n}=\mathbf{1 6 7})\end{array}$ & $\begin{array}{c}\text { Depression } \\
(\boldsymbol{n}=\mathbf{1 2 8})\end{array}$ & $\boldsymbol{P}^{\text {-value }^{\mathrm{c}}}$ \\
\hline Sex (male/female) (no.) & $56 / 111$ & $34 / 94$ & 0.19 \\
Age [mean (SD) years] & $50.4(13)$ & $54.6(12)$ & 0.04 \\
High educational level (\%) & 30.6 & 18.7 & 0.03 \\
Married (\%) & 92.9 & 97.4 & 0.04 \\
Low income level (\%) & 52.4 & 64.1 & 0.03 \\
Overweight (\%) & 79.9 & 76.5 & 0.09 \\
Current smoker (\%) & 9.0 & 11.8 & 0.48 \\
Duration of diabetes [mean (SD) years] & $7.8(5.1)$ & $9.2(6.8)$ & 0.03 \\
Complications of diabetes (1+) (\%) & 46.2 & 55.6 & 0.04 \\
Treatment intensity (insulin therapy) (\%) & 19.4 & 14.6 & 0.34 \\
\hline
\end{tabular}

${ }^{a} B D I$ score $<15 ;{ }^{b} B D I$ score $\geq 15 ; \chi^{2} \chi^{2}$ test or t-test.

$S D=$ standard deviation. 


\begin{tabular}{|c|c|c|c|c|}
\hline Variable & $\begin{array}{c}\text { Men } \\
(n=34)\end{array}$ & $\begin{array}{l}\text { Women } \\
(n=94)\end{array}$ & OR $(95 \% \mathrm{CI})$ & $P$-value \\
\hline Age [mean (SD) years] & $55.7(16.9)$ & $54.2(12.4)$ & - & 0.88 \\
\hline Family history of diabetes (\%) & 47.1 & 38.3 & $1.43(0.64-3.10)$ & 0.19 \\
\hline High educational levela $(\%)$ & 38.2 & 11.7 & $4.20(1.10-5.60)$ & $<0.0001$ \\
\hline Married (\%) & 91.2 & 98.8 & $1.50(1.30-3.10)$ & 0.04 \\
\hline Low income level (\%) & 51.5 & 64.9 & $0.57(0.20-1.20)$ & 0.06 \\
\hline Overweight ${ }^{\mathrm{b}}(\%)$ & 72.7 & 87.1 & $2.35(1.95-6.70)$ & $<0.0001$ \\
\hline Current smoker (\%) & 30.0 & 4.3 & $9.40(2.10-11.30)$ & $<0.0001$ \\
\hline Duration of diabetes [mean (SD) years] & $9.8(6.8)$ & $8.9(6.7)$ & - & 0.84 \\
\hline Complications of diabetes (\%) & 44.1 & 44.7 & $0.97(0.44-2.10)$ & 0.88 \\
\hline Treatment intensity (insulin therapy) (\%) & 14.5 & 14.8 & $0.90(0.70-1.30)$ & 0.73 \\
\hline
\end{tabular}

${ }^{a}$ High school and more; ${ }^{b} B M I=$ body mass index $\geq 25 \mathrm{~kg} / \mathrm{m}^{2}$

$S D=$ standard deviation; $O R=$ odds ratio; $C I=$ confidence interval. .

Female patients with depression were more likely to be overweight (OR 2.35, 95\% CI: $1.95-6.70)$ and to be married (OR 1.50, 95\% CI: 1.30-3.10) compared with men. Male patients were more to have a high educational level (OR 4.20, 95\% CI: 1.10-5.60) than women. Other variables, e.g. family history of diabetes, complications, low income, duration of diabetes, treatment intensity and age, had no relationship with the sex of depressed patients ( $\mathrm{Ta}$ ble 2).

Although depressed men and women had a similar prevalence of complications, there were sex differences in the relationship between complications and depression, and the association was statistically significant for women but not for men. Comparing women with depression with nondepressed women, older age (> 50 years) $(58.2 \%$ versus $50.5 \%$ ), having complications (55.3\% versus $40.5 \%$ ), being unmarried ( $92.8 \%$ versus $98.9 \%$ ) and duration of diabetes [mean 7.3 (SD 5.2) versus 8.9 (SD 6.1) years] had a significant effect on depression $(P<0.05)$. Twice as many women patients with depression had 2 or more complications of diabetes (12.0\%) compared with women with no depression (6.5\%). However, we could find no relation between other behavioural and clinical factors among female subjects. Low income was the only variable that influenced having depression in diabetic men; $32.1 \%$ nondepressed versus depressed $51.5 \%$ men had a low income).

Using logistic regression analysis, entering all confounder variable in the model, older age was the only variable that remained significantly related to depression $(\mathrm{OR}=2.02,95 \% \mathrm{CI}: 1.52-$ 3.80, $\exp (\mathrm{B})=1.023, P<0.01$, constant: $-1.49)$.

\section{Discussion}

In view of the increasing prevalence of diabetes in developing countries and its association with depression, the detection and control of depression symptoms has some benefits in the management of patients with diabetes.

The overall prevalence of depression in our setting, based on BDI scores $\geq 15$, was $43.4 \%$. Severe depression was detected in only $1.4 \%$ of patients. The overall frequency of elevated depression symptoms was quite high compared with studies in other countries, where investigators found elevated depression scores on the BDI in $26 \%$ of diabetic patients $[7,14]$. However, a similar frequency of depression as our study was reported in other studies in the Islamic Republic of Iran [15-17].

Epidemiological studies of psychiatric disorders in the general population in the Islamic Republic of Iran are limited and the prevalence varies across different studies. A survey in west Azerbaijan reported the prevalence of psychiatric disorders as $29.0 \%$ (40.2\% in women and $17.7 \%$ in men), anxiety $14.0 \%$ and mood disorders $8 \%$ [18]. Another epidemiological assessment of mental disorders among people aged 15 years or older in urban Natanz showed that the most prevalent disorders were dysthymia (5.8\%), generalized anxiety (5.3\%) and depression (3.3\%) [19]. In Hamedan, $8.1 \%$ of studied individuals suffered from at least one psychiatric disorder, of which $2.6 \%$ had major depression [20]. Anxiety and mood disorders were the most prevalent psychiatric disorders in east Azarbaijan province (8.5\% and 2.9\%) [21]. Trends in perceptions of depression may be partly attributable to cultural attitudes toward depression $[7,10]$.

In our study, diabetic patients with depression scores on the BDI had fewer years of education, lower income and were older than those who did not. Indeed financial stress and social support may contribute the most to depression 
among diabetic patients. These findings agree with studies which found that the frequency of depression was associated with social and financial factors [2,22]. Age was the only variable related to depression in our multiple regression model. The greater risk of depression in older versus younger patients may be because older patients have more problems in coping physically and psychologically with a greater number of diabetes complications.

An association between disease duration and depression was revealed in our study. In contrast, Fisher et al. found no relation between length of diabetes and depression [23]. Other studies showed that disease duration was significantly correlated with depression scores [15-17,22,24]. Meanwhile, treatment type had only a weak influence on depression severity in some studies $[17,25,26]$ and the intensity of therapy (insulin or other) had no effect on depression in our study. Patients with complications of diabetes also had higher depression scores in our study. Patients with complications typically require long-term medical care and are consequently suffer more psychological stress; therefore, increased attention should be given to these cases [25].

A gender effect on the frequency of depression has been documented for several conditions [14]. In our study the prevalence of elevated depression symptoms was the same in women and men but the mean BDI score was significantly higher in women. Although some studies found that women with diabetes experience more psychological distress than men $[16,17,25]$, a meta-analysis revealed that the increased odds of depression associated with diabetes according to sex were about the same [7].

BMI had no effect on depression score in our diabetic patients. This is in contrast to Robinson et al. and Gavard et al. who reported that currently depressed diabetic individuals had a significantly higher BMI than nondepressed diabetics [26]. However, the frequency of BMI $\geq 25 \mathrm{~kg} / \mathrm{m}^{2}$ was high in our study sample and significantly more depressed women had high BMI than did depressed men. The combination of depression and obesity may worsen the course of diabetes because both factors are associated with an increased risk of adverse cardiac outcomes.
One limitation of the study was the use of self-reported questionnaires instead of psychiatric interviews. Many studies, however, have used the same methods. Another limitation in our study was to evaluate education and income based on patients' self-reporting. Despite these limitations the results are valuable in that they confirm the importance of age as an associated variable in depression in diabetic patients [27].

In conclusion, the high prevalence of depression in diabetes patients in Urmia is an important finding, as depression has a negative impact on quality of life. Depression has additional importance in diabetes because of its association with poor compliance with diabetes treatment, poor glycaemic control and an increased risk of micro- and macrovascular disease complications [2]. People with diabetes and depression have greater use of emergency care, outpatient primary care, medical and psychiatric specialty care, medical inpatient care and higher prescription costs [28]. This will become an important consideration as the prevalence of diabetes is estimated to rise in Asia with a corresponding increase in the economic burden of the disease $[10,29,30]$.

\section{References}

1. Brown LC et al. History of depression increases risk of type 2 diabetes in younger adults. Diabetes care, 2005, 28(5):1063-7.

2. Katon $\mathrm{W}$ et al. Behavioral and clinical factors associated with depression among individuals with diabetes. Diabetes care, 2004, 27(4):914-20.

3. Lustman PJ, Clouse RE. Depression in diabetic patients: the relationship between mood and glycemic control. Journal of diabetes and its complications, 2005, 19(2):113-22.

4. Katon WJ et al. The association of comorbid depression with mortality in patients with type 2 diabetes. Diabetes care, 2005, 28(11):2668-72.

5. Bruce DG et al. A prospective study of depression and mortality in patients with type 2 diabetes: the Fremantle Diabetes Study. Diabetologia, 2005, 48(12):2532-9.

6. Katon W et al. Cost-effectiveness and net benefit of enhanced treatment of depression for older adults with diabetes and depression. Diabetes care, 2006, 29(2):265-70.

7. Anderson RJ et al. The prevalence of comorbid depression in adults with diabetes: a meta-analysis. Diabetes care, 2001, 24(6):1069-78
8. De Groot $\mathrm{M}$ et al. Depression treatment and satisfaction in a multicultural sample of type 1 and type 2 diabetic patients. Diabetes care, 2006, 29(3):549-53.

9. Eaton WW et al. Depression and risk for onset of type II diabetes. A prospective population-based study. Diabetes care, 1996, 19(10):1097-102.

10. Zahid $\mathrm{N}$ et al. Depression and diabetes in a rural community in Pakistan. Diabetes research and clinical practice, 2007, 79(1):124-7.

11. King H, Aubert RE, Herman WH. Global burden of diabetes, 1995-2025: prevalence, numerical estimates, and projections. Diabetes care, 1998, 21(9):1414-31.

12. Murray CJL, Lopez AD, eds. The Global Burden of Disease. A comprehensive assessment of mortality and disability from diseases, injuries and risk factors in 1990 and projected to 2020. Cambridge, Massachusetts, Harvard School of Public Health on behalf of the World Health Organization and the World Bank, 1996.

13. Kaviani H, Musavi A, Mohit A. [Psychological interviews and scales.] Tehran, Islamic Republic of Iran, SANA Publishers, 2001 [in Farsi]. 
14. Tellez-Zenteno JF, Cardiel MH. Risk factors associated with depression in patients with type 2 diabetes mellitus. Archives of medical research, 2002, 33(1):53-60.

15. Taziki A et al. [The association between depression and diabetes.] Journal of Gorgan University of Medical Sciences, 2001, 8:59-64 [in Farsi].

16. Sepehrmanesh $Z$ et al. [The prevalence of depression and its related factors among diabetic patients.] Journal of Kashan University of Medical Sciences, 2002, 7:69-75 [in Farsi].

17. Larijani B et al. [The association between depression and diabetes among diabetic patients in Shariati hospital.] Iranian journal of diabetes and lipid disorders, 2003, 3(1):77-82 [in Farsi].

18. Mohammadi MR et al. [An epidemiological study of psychiatric disorders in west Azerbaijan province.] Urmia medical journal, 2004, 14(4):242-52 [in Farsi].

19. Omidi ATA, Sazvar SA, Akkasheh G. [Epidemiology of mental disorders in urbanized areas of Natanz.] Iranian journal of psychiatry and clinical psychology, 2001, 8(4):32-8 [in Farsi].

20. Mohammadi MR et al. [An epidemiological study of psychiatric disorders in Hamadan province, 2001.] Scientific Journal of Hamadan University of Medical Sciences and Health Services, 2004 11(3):28-36 [in Farsi]

21. Mohammadi MR et al. [An epidemiological study of psychiatric disorders in east Azerbaijan province.] Medical Journal of Tabriz University of Medical Sciences and Health Services, 2005, 64:67-73 [in Farsi].
22. Musselman DL et al. Relationship of depression to diabetes types 1 and 2: epidemiology, biology, and treatment. Biological psychiatry, 2003, 54(3):317-29.

23. Fisher $\mathrm{L}$ et al. Contributors to depression in Latino and European-American patients with type 2 diabetes. Diabetes care, 2001, 24(10):1751-7.

24. Starostina EG. Depression and anxiety in type 2 diabetes in relation to disease characteristics and psychosocial variables: a pilot assessment. Journal of psychosomatic research, 2006, 60(6):663.

25. Bai YL et al. Correlates of depression in type 2 diabetic elderly patients: a correlational study. International journal of nursing studies, 2008, 45(4):571-9.

26. Sevincok $L$ et al. Depression in a sample of Turkish type 2 diabetes patients. European psychiatry, 2001, 16(4):229-31.

27. Gilmer TP et al. Predictors of health care costs in adults with diabetes. Diabetes care, 2005, 28(1):59-64.

28. Zhao $\mathrm{W}$ et al. Association between diabetes and depression sex and age differences. Public health, 2006, 120(8):696-704.

29. Simon GE et al. Diabetes complications and depression as predictors of health service costs. General hospital psychiatry, 2005, 27(5):344-51

30. Shah ZC, Huffman FG. Depression among Hispanic women with type 2 diabetes. Ethnicity and disease, 2005, 15(4):685-90.

\section{Mental health systems in selected low-and middle-income countries}

Mental health systems in selected low-and middle-income countries summarizes descriptive data on mental health systems of selected low- and middle-income countries (LAMICs) using the World Health Organization Assessment Instrument for Mental health Systems (WHO-AIMS).

The comprehensive and detailed information gathered through WHO-AIMS and summarized in this report provides a better understanding of mental health systems in these countries. Results indicate that mental health resources and activities are scarce, inequitably distributed and inefficiently used; community-based mental health services are underdeveloped; mental health systems are often not well connected to other relevant sectors, such as the primary health care system; and that insufficient attention is given to human rights.

This report highlights the urgent need for additional resources, and the importance of ensuring better use of the limited resources available. The information derived from this WHO-AIMS study is being used to develop plans for strengthening community care and scaling up services for people with mental disorders.

Further information about this and other WHO publications can be found at: http://www.who.int/publications/en/ 